\title{
THE SPECTRUM AND COMMUTANT \\ OF A CERTAIN WEIGHTED TRANSLATION OPERATOR
}

\author{
KARL PETERSEN*
}

\section{Introduction.}

A weighted translation operator is an operator on $L^{2}(X, \mathscr{B}, \mu)$, for some measure space $(X, \mathscr{B}, \mu)$, of the form

$$
S f(x)=\varphi(x) f(\tau x) \quad\left(f \in L^{2}(X, \mathscr{B}, \mu)\right),
$$

where $\varphi \in L^{\infty}(X, \mathscr{B}, \mu)$ and $\tau: X \rightarrow X$ is a measure-preserving transformation (this means that $\tau^{-1} \mathscr{B} \subset \mathscr{B}$ and $\mu\left(\tau^{-1} A\right)=\mu(A)$ for all $A \in \mathscr{B}$ ). The class of such operators has been studied by Parrott [7] and Bastian [1] and includes all the weighted shifts. We consider the particular weighted translation operator $T$ defined on $L^{2}[0,1)$ by letting

$$
\varphi(x)=r^{x_{[0, \beta)(x)}}
$$

for some fixed $r>0$ and $\beta \in[0,1)$ and $\tau(x)=\langle x+\alpha\rangle$ for some irrational $\alpha$, where $\langle y\rangle$ denotes the fractional part of a real number $y$; thus

$$
T f(x)=r^{\chi_{[0, \beta)}(x)} f\langle x+\alpha\rangle \quad\left(f \in L^{2}[0,1)\right) .
$$

The operator $T$ was investigated previously, in the case $\beta=\alpha$, by Rudin (unpublished notes), who showed that then the eigenfunctions of $T$ span $L^{2}[0,1)$ and hence $T$ is similar to a normal operator. He also proved that, writing

$$
T_{r} f(x)=r^{x_{[0, \alpha)}(x)} f\langle x+\alpha\rangle \quad\left(f \in L^{2}[0,1)\right),
$$

if $r_{1} \neq r_{2}$ then for each nonzero $f \in L^{2}[0,1)$ the linear span of

$$
\left\{T_{r_{1}}^{n} f: n \geqq 0\right\} \cup\left\{T_{r_{2}}^{n} f: n \geqq 0\right\}
$$

is dense in $L^{2}[0,1)$. Thus examples of this type, which are easier to work with than, for example, the Bishop operators

$$
B_{\alpha} f(x)=x f\langle x+\alpha\rangle \quad\left(f \in L^{2}[0,1)\right),
$$

may be of interest in relation to the invariant subspace problem. Howe-

* Research supported in part by N.S.F. Grant GP-42657.

Received February 18, 1975. 
ver, just as Davie [2] has found nontrivial invariant subspaces for $B_{\alpha}$ for almost every $\alpha$, so it follows already from results of Wermer [11] and Khintchine [6] that our operator $T$ has a nontrivial invariant subspace at least for almost every $\alpha$. (On the other hand, Parrott [7] has noted that $T$ never has a nontrivial reducing subspace.)

Nevertheless, the operator $T$ has several interesting properties, perhaps the most striking of which are the radical differences in its spectrum and commutant depending on whether or not $\beta \in \mathbf{Z}_{\alpha}(\bmod 1)$ (that is, whether or not $\beta=\langle n \alpha\rangle$ for some $n \in Z$ Z). In either case the spectrum of $T$ is $\left\{z:|z|=r^{\beta}\right\}$ (Proposition 2.2), but $T$ has nonzero measurable eigenfunctions if and only if $\beta \in Z_{\alpha}(\bmod 1)$ (Theorem 2.3). Generalizing the case when $\beta=\alpha$, if $\beta \in \mathrm{Z}_{\alpha}(\bmod 1)$ then the eigenfunctions of $T$ span $L^{2}[0,1)$ and $T$ is similar to a normal operator (Theorem 2.4). When $\beta \in \mathrm{Z} \alpha(\bmod 1)$, the commutant of $T$ can be characterized (Theorem 3.1) and contains many weighted translation operators; but if $\beta \notin Z_{\alpha}(\bmod 1)$, then the only invertible weighted translation operators that commute with $T$ are multiples of the powers of $T$ (Theorem 3.2). Our final observations indicate some possible applications of this line of thought to a problem in diophantine approximation.

I am grateful to J. Stampfli for interesting me in these matters, to W. Rudin for apprising me of his work on the operator $T$, and to $J$. Cima and W. Wogen for their contributions to this investigation.

\section{The spectrum.}

In order to identify the spectrum of $T$ we make use of the following results, due (in a slightly different form) to Parrott [7], on more general weighted translation operators. Recall that an invertible (that is, oneto-one onto a.e. with measurable inverse) measure-preserving transformation $\tau$ is said to be ergodic if every measurable set $A$ with $\tau A \subset A$ has measure 0 or 1 . For example, $\tau x=\langle x+\alpha\rangle$ is ergodic when $\alpha$ is irrational. It is known that $\tau$ is ergodic if and only if every $\tau$-invariant (that is, $f \circ \tau=f$ a.e. measurable function is constant a.e.

Theorem 2.1. Let $\tau:[0,1) \rightarrow[0,1)$ be an ergodic measure-preserving transformation, $\varphi \in L^{\infty}[0,1)$, and $S f(x)=\varphi(x) f(\tau x)$ for $f \in L^{2}[0,1)$.

(1) If $\varphi_{n}(x)=\varphi(x) \varphi(\tau x) \ldots \varphi\left(\tau^{n-1} x\right)$ for $n=1,2, \ldots$, then

$$
\lim _{n \rightarrow \infty}\left|\varphi_{n}(x)\right|^{1 / n}=\exp \int \log |\varphi| \text { a.e. }
$$

(2) The spectral radius $r(S)$ of $S$ satisfies $r(S) \geqq \exp \int \log |\varphi|$. 
(3) The spectrum of $S$ is closed under rotation: $e^{i \theta} \sigma(S) \subset \sigma(S)$ for each $\theta \in[0,2 \pi)$.

Proof. (1) If $\log |\varphi| \in L^{1}[0,1)$, then by the Ergodic Theorem

$$
\lim _{n \rightarrow \infty} n^{-1} \sum_{k=0}^{n-1} \log \left|\varphi\left(\tau^{k} x\right)\right|=\Phi(x)
$$

exists a.e., and, since $\tau$ is ergodic, $\Phi(x)=\int \log |\varphi|$ a.e. Then the result follows upon exponentiation. If $\log |\varphi| \notin L^{1}$, then $\int(\log |\varphi|)^{-}=\infty$ and a standard argument $[4$, p. 32], shows that

hence

$$
\lim _{n \rightarrow \infty} n^{-1} \sum_{k=0}^{n-1} \log \left|\varphi\left(\tau^{k} x\right)\right|=\infty \quad \text { a.e.; }
$$

$$
\lim _{n \rightarrow \infty}\left|\varphi_{n}(x)\right|^{1 / n}=0=\exp \int \log |\varphi| \quad \text { a.e. }
$$

(2) Since the spectral radius of $S$ is given by $r(S)=\lim _{n \rightarrow \infty}\left\|S^{n}\right\|^{1 / n}$ and $\left\|S^{n}\right\|=\left\|\varphi_{n}\right\|_{\infty}$, this statement is an immediate consequence of (1).

(3) Denote by $\Pi(R)$ the set of approximate eigenvalues of an operator $R$ on $L^{2}[0,1)$; that is, $\Pi(R)$ is the set of all those $\lambda \in \mathrm{C}$ for which given any $\varepsilon>0$ there may be found an $f \in L^{2}[0,1)$ with

$$
\|f\|_{2}=1 \text { and }\|R f-\lambda f\|_{2}<\varepsilon .
$$

Define $U_{\tau}$ on $L^{2}[0,1)$ by $U_{\tau} f(x)=f(\tau x)$. We will show first that

$$
\Pi\left(U_{\tau}\right)=\mathrm{K}=\{z:|z|=1\}
$$

and $\pi(S) \Pi\left(U_{\tau}\right) \subset \Pi(S)$.

To see that $\Pi\left(U_{\tau}\right)=\mathrm{K}$, let $\varepsilon>0$ and $\lambda \in \mathrm{K}$ be given. A result of Rokhlin [9] and Kakutani [5] (see also [4, p. 71]) allows us to choose an integer $n>8 / \varepsilon^{2}$ and a measurable set $E$ with $E, \tau E, \ldots, \tau^{n-1} E$ pairwise disjoint and

$$
\mu\left(\bigcup_{k=0}^{n-1} \tau^{k} E\right) \geqq 1-\varepsilon^{2} / 8 .
$$

We let $f_{0}(x)=\lambda^{k}$ on $\tau^{k} E$ for $k=0, \ldots, n-1$ and $f_{0}(x)=1$ if $x \in[0,1) \backslash$ $\bigcup_{k=0}^{n-1} \tau^{k} E$. Then $\left|f_{0}\right|=1,\left\|f_{0}\right\|_{2}=1$, and $f_{0} \circ \tau=\lambda f_{0}$ on $E \cup \tau E \cup \ldots \cup \tau^{n-2} E$; consequently

$$
\begin{gathered}
\left\|\lambda f_{0}-U_{\tau} f_{0}\right\|_{2}^{2}=\int_{[0,1) \backslash U_{0 \leqq k \leqq n-2} \tau^{k} E}\left|\lambda f_{0}(x)-f_{0}(\tau x)\right|^{2} d x \\
\leqq 4\left[\mu(E)+\frac{1}{8} \varepsilon^{2}\right] \leqq 4\left[n^{-1}+\frac{1}{8} \varepsilon^{2}\right]<\varepsilon^{2} .
\end{gathered}
$$

Now let $\varrho \in \Pi(S), \lambda \in \Pi\left(U_{\tau}\right)$, and $\varepsilon>0$. Choose $g_{0} \in L^{2}[0,1)$ with $\left\|\varrho g_{0}-S f_{0}\right\|_{2}<\frac{1}{4} \varepsilon$ and $g \in L^{\infty}[0,1)$ with

Then

$$
\left\|g-g_{0}\right\|_{2}<\varepsilon / 4(\|S\|+|\varrho|+1) .
$$




$$
\|S g-\varrho g\|_{2} \leqq\left\|S g_{0}-\varrho g_{0}\right\|_{2}+\left\|g-g_{0}\right\|_{2}(\|S\|+|\varrho|)<\frac{1}{2} \varepsilon .
$$

Let $\delta=\varepsilon /\left(1+\|S g\|_{\infty}\right)$ and as before choose an integer $n \geqq 32 \delta^{-2}$ and a measurable set $E$ with $E, \tau E, \ldots, \tau^{n-1} E$ pairwise disjoint and

$$
\mu\left(\bigcup_{k=0}^{n-1} \tau^{k} E\right) \geqq 1-\delta^{2} / 32 .
$$

Let $h(x)=\lambda^{k}$ on $\tau^{k} E$ for $k=0, \ldots, n-1$ and $h(x)=1$ otherwise. Then

and

$$
\|h\|_{2}=1, \quad\left\|\lambda h-U_{\tau} h\right\|_{2}<\frac{1}{2} \delta,
$$

Hence $\varrho \lambda \in \Pi(S)$.

$$
\begin{aligned}
& \|S(g h)-\varrho \lambda g h\|_{2}=\left\|\left(U_{\tau} h\right) S g-\varrho \lambda g h\right\|_{2} \\
& \quad=\left\|\left(U_{\tau} h-\lambda h\right) S g+\lambda h(S g-\varrho g)\right\|_{2} \\
& \quad \leqq\|S g\|_{\infty}\left\|U_{\tau} h-\lambda h\right\|_{2}+\|S g-\varrho g\|_{2}<\varepsilon .
\end{aligned}
$$

Since the adjoint $S^{*}$ of $S$ is given by

$$
S^{*} f(x)=\bar{\varphi}\left(\tau^{-1} x\right) f\left(\tau^{-1} x\right) \quad\left(f \in L^{2}[0,1)\right),
$$

the preceding argument may be repeated to show that $\Pi\left(S^{*}\right) \Pi\left(U_{\tau-1}\right) \subset$ $\Pi\left(S^{*}\right)$. Therefore

$$
\begin{aligned}
\sigma(S) & =\Pi(S) \cup\left(\Pi\left(S^{*}\right)\right)^{-} \supset\left[\Pi(S) \Pi\left(U_{\tau}\right)\right] \cup\left[\Pi\left(S^{*}\right) \Pi\left(U_{\tau}\right)\right] \\
& =[\Pi(S) \mathrm{K}] \cup\left[\left(\Pi\left(S^{*}\right)\right)^{-} \mathrm{K}\right]=\left[\Pi(S) \cup\left(\Pi\left(S^{*}\right)\right)^{-}\right] \mathrm{K}=\sigma(S) \mathrm{K},
\end{aligned}
$$

where the bar denotes complex conjugation.

We turn our attention now to the operator

$$
T f(x)=r^{x_{[0, \beta)(x)}} f\langle x+\alpha\rangle \quad\left(f \in L^{2}[0,1)\right) .
$$

$T$ is invertible with inverse

If we let

$$
T^{-1} f(x)=r^{-x_{[0, \beta)}\langle x-\alpha\rangle} f\langle x-\alpha\rangle \quad\left(f \in L^{2}[0,1)\right) .
$$

$$
u_{n}(x)=\sum_{k=0}^{n-1} \chi_{[0, \beta)}\langle x+k \alpha\rangle
$$

for $n=1,2, \ldots$, then $\varphi_{n}(x)=r^{u_{n}(x)}$ and, because $\{\langle k \alpha\rangle: k=0,1,2, \ldots\}$ is equidistributed $\bmod 1$,

$$
\lim _{n \rightarrow \infty}\left|\varphi_{n}(x)\right|^{1 / n}=r^{\beta} \quad \text { for all } x \in[0,1) .
$$

Proposition 2.2. The spectrum of $T$ is $\sigma(T)=\left\{z \in \mathrm{C}:|z|=r^{\beta}\right\}$.

Proof. From Theorem 2.1 (2) it follows that $r(T) \geqq r^{\beta}$. By an elementary result in number theory, there are infinitely many pairs of relatively prime positive integers $p$ and $q$ with

$$
|\alpha-p / q|<q^{-2}
$$


choose one such pair. The points $\langle-k \alpha\rangle, 0 \leqq k \leqq q-1$, are spread throughout the unit interval with spacing smaller than $2 / q$, and $u_{q}(x)$ counts the number of these points that lie in the translate by $x$ of the interval $[0, \beta)$. Therefore, if $k / q \leqq \beta<(k+1) / q$ we must have $k-1 \leqq u_{q}(x) \leqq k+2$, and so $\left|u_{q}(x)-u_{q}(y)\right| \leqq 3$ for all $x, y \in[0,1)$. Thus

and

$$
\left|\varphi_{q}(x)\right|=r^{u_{q}(x)} \leqq r^{u_{q}(0) \pm 3} \quad(x \in[0,1))
$$

$$
r(T)=\lim _{q \rightarrow \infty}\left\|T^{q}\right\|^{1 / q} \leqq \lim _{q \rightarrow \infty} r^{\left[u_{q}(0) \pm 3\right] / q}=r^{\beta} .
$$

A similar argument applied to $T^{-1}$ shows that $r\left(T^{-1}\right)=r^{-\beta}$, and this implies that $\sigma(T) \subset\left\{z:|z| \geqq r^{\beta}\right\}$, so in fact we must have $\sigma(T) \subset\left\{z:|z|=r^{\beta}\right\}$. The result then follows from Theorem 2.1 (3).

Theorem 2.3. T has a nonzero measurable eigenfunction if and only if $\beta \in \mathrm{Z}_{\alpha}(\bmod 1)$.

Proof. If $\beta \in Z_{\alpha}(\bmod 1)$, say $\beta=\langle n \alpha\rangle$ for some nonzero integer $n$, let

$$
\psi(x)=\left\{\begin{array}{cl}
\langle x-\alpha\rangle+\ldots+\langle x-n \alpha\rangle & \text { if } n>0 \\
-\langle x\rangle-\ldots-\langle x-(n+1) \alpha\rangle & \text { if } n<0 .
\end{array}\right.
$$

(If $\beta=0, \mathrm{e}^{2 n i k x}$ is an eigenfunction of $T$ for each $k \in \mathrm{Z}$.) Since

we have

$$
\langle x-\beta\rangle-\langle x\rangle=\chi_{[0, \beta)}(x)-\beta \text {, }
$$

$$
\psi(x)-\psi\langle x+\alpha\rangle=\chi_{[0, \beta)}(x)-\beta,
$$

and hence $r^{\psi(x)}$ is an eigenfunction of $T$ with eigenvalue $r^{\beta}$.

Conversely, suppose that there is $\lambda \in \mathrm{C}$ and a nonzero measurable function $f$ with $T f(x)=\lambda f(x)$ a.e. By Proposition $2.2,|\lambda|=r^{\beta}$; by taking absolute values we may assume that $\lambda=r^{\beta}$ and $f(x) \geqq 0$ a.e. Since $\{x$ : $f(x)=0\}$ is invariant under translation by $\alpha$ and hence has measure 0 , we may define

then, since

$$
\xi(x)=\exp \left[2 \pi i \frac{\log f(x)}{\log r}\right]
$$

$$
\begin{gathered}
r^{\chi_{[0, \beta)(x)}} f\langle x+\alpha\rangle=r^{\beta} f(x) \quad \text { a.e. , } \\
\log f\langle x+\alpha\rangle=\left[\beta-\chi_{[0, \beta)}(x)\right] \log r+\log f(x),
\end{gathered}
$$

and

$$
\xi\langle x+\alpha\rangle=\exp \left[2 \pi i\left(\beta-\chi_{[0, \beta)}(x)\right)+\frac{\log f(x)}{\log r}\right]=e^{2 \pi i \beta} \xi(x),
$$

and this implies that $\beta \in Z_{\alpha}(\bmod 1)$. 
Theorem 2.4. If $\beta \in \mathrm{Z}_{\alpha}(\bmod 1)$, then every eigenvalue of $T$ is simple, every eigenfunction of $T$ is a constant multiple of one of the functions

$$
f_{k}(x)=e^{2 \pi i k x} r^{\psi(x)}
$$

for some $k \in \mathrm{Z}$ (where $\psi$ is as in the proof of Theorem 2.3), the eigenfunctions of $T$ span $L^{2}[0,1)$, and $T$ is similar to a constant multiple of the unitary operator $U_{\alpha}$ defined on $L^{2}[0,1)$ by $U_{\alpha} f(x)=f\langle x+\alpha\rangle$.

Proof. It is easily verified that each $f_{k}(x)$ is an eigenfunction of $T$ with eigenvalue $e^{2 \pi i k \alpha} r^{\beta}$. Now if $T f=\lambda f$ and $T g=\lambda g$ a.e. for some nonzero measurable $f$ and $g$, then, noting that $\{x: g(x)=0\}$ is invariant under translation by $\alpha$ and hence has measure 0 , we see that

$$
\frac{f\langle x+\alpha\rangle}{g\langle x+\alpha\rangle}=\frac{f(x)}{g(x)} \quad \text { a.e. , }
$$

and hence $f / g$ is constant a.e. This shows that every eigenvalue is simple.

To show that every eigenfunction is a multiple of some $f_{k}$, it suffices now to prove that the argument of every eigenvalue of $T$ is a multiple of $\alpha \bmod 1$. But if $T f=\lambda f$ and $\lambda=r^{\beta} e^{2 \pi i \theta}$, then from the equation

we find that

$$
r^{x_{[0, \beta)}(x)} f\langle x+\alpha\rangle=r^{\beta} e^{2 \pi i \theta} f(x)
$$

$$
e^{2 \pi i \arg f\langle x+\alpha\rangle}=e^{2 \pi i \theta} e^{2 \pi i \arg f(x)},
$$

and hence $\theta \in Z_{\alpha}(\bmod 1)$.

If $h \in L^{2}[0,1)$ and $h$ is orthogonal to all the functions $f_{k}, k \in Z$, then $h r^{\psi(x)}=0$ a.e. and hence $h=0$ a.e. Thus the eigenfunctions of $T$ span $L^{2}[0,1)$.

Finally, if we let $S f(x)=r^{\varphi(x)} f(x)$ for $f \in L^{2}[0,1)$, then $S$ is invertible and, because of (A),

$$
S^{-1} T S f(x)=r^{\beta} f\langle x+\alpha\rangle=r^{\beta} U_{\alpha} f(x) .
$$

\section{The commutant.}

In this section we seek information about operators $S$ on $L^{2}[0,1)$ that commute with the operator $T f(x)=r^{\chi_{[0, \beta)(x)}} f\langle x+\alpha\rangle$. In case $S$ is a weighted translation operator, say $S f(x)=\varphi(x) f(\tau x)$ with $\varphi \in L^{\infty}[0,1)$ and $\tau$ a measure-preserving transformation on $[0,1)$, the condition $T S=S T$ says that

$$
\begin{array}{r}
\varphi(x) r^{\chi_{[0, \beta)(\tau x)}} f\langle\tau x+\alpha\rangle=r_{[0, \beta)}^{\chi_{[0}(x)} \varphi\langle x+\alpha\rangle f(\tau\langle x+\alpha\rangle) \\
\text { a.e. }\left(f \in L^{2}[0,1)\right),
\end{array}
$$


and this implies, upon taking $f \equiv 1$, that

$$
\varphi(x) r^{\alpha_{[0, \beta)(\tau x)}}=r^{\chi_{[0, \beta)(x)}} \varphi\langle x+\alpha\rangle \quad \text { a.e. , }
$$

and hence $\langle\tau(x)+\alpha\rangle=\tau\langle x+\alpha\rangle$ a.e. From the latter statement it follows that there is $\gamma \in[0,1)$ such that $\tau x=\langle x+\gamma\rangle$ a.e. If

then

$$
\eta(x)=\log |\varphi(x)| / \log r,
$$

$$
\eta(x)-\eta\langle x+\alpha\rangle=\chi_{[0, \beta)}(x)-\chi_{[0, \beta)}\langle x+\gamma\rangle \quad \text { a.e. }
$$

Theorem 3.1. Suppose $\beta=\langle n \alpha\rangle$ and let $f_{k}(x)=e^{2 \pi i k x} r^{\psi(x)}$ for $k \in \mathbf{Z}$.

(1) If $S T=T S$, then there are constants $c_{k}, k \in \mathrm{Z}$, with $S f_{k}=c_{k} f_{k}$ and $\left|c_{k}\right| \leqq\|S\|$ for all $k$.

(2) Conversely, given a bounded sequence $\left\{c_{k}: k \in \mathbf{Z}\right\}$, the equations $S f_{k}=c_{k} f_{k}(k \in \mathrm{Z})$ define a bounded operator $S$ which is a strong limit of polynomials in $T$ and hence commutes with $T$.

(3) If $S T=T S$ and $S f(x)=\varphi(x) f(\tau x)\left(f \in L^{2}[0,1)\right)$ for some $\varphi \in L^{\infty}[0,1)$ and some measure-preserving $\tau$ on $[0,1)$, then are constants $c \in \mathrm{C}$ and $\gamma \in[0,1)$ such that $\tau x=\langle x+\gamma\rangle$ a.e. and

$$
\varphi(x)=c r^{\psi(x)-\psi\langle x+\gamma\rangle} \quad \text { a.e. }
$$

(4) Conversely, given any $c \in \mathrm{C}$ and $\gamma \in[0,1)$, let

and

$$
\varphi(x)=c r^{\psi(x)-\psi\langle x+\gamma\rangle}
$$

$$
S f(x)=\varphi(x) f\langle x+\gamma\rangle \quad\left(f \in L^{2}[0,1)\right) .
$$

Then $S T=T S$ and

$$
S f_{k}=c e^{2 \pi i k v} f_{k} \quad \text { for all } k
$$

Proof. Since $T$ is similar to $r^{\beta} U_{\alpha}$, (1) and (2) follow from known results on the commutants of diagonal operators. However, we include proofs of these statements for the sake of completeness.

(1) It is easy to check that for $k \in Z S f_{k}$ is an eigenfunction of $T$ with eigenvalue $e^{2 \pi i k \alpha} r^{\beta}$. Since each eigenvalue is simple, $S f_{k}=c_{k} f_{k}$ for some $c_{k} \in \mathrm{C}$, and clearly $\left|c_{k}\right| \leqq\|S\|$ for all $k$.

(2) If we let $d \mu(x)=r^{-2 \psi(x)} d x$, then in $L^{2}(\mu)$ the functions $f_{k}$ form a complete orthonormal set, and

$$
r^{-n}\|f\|_{2} \leqq\|f\|_{L^{2}(\mu)} \leqq r^{n}\|f\|_{2}
$$

for each measurable function $f$ on $[0,1]$. The equations $S f_{k}=c_{k} f_{k}$ define a 
bounded linear operator of norm $\sup _{k}\left|c_{k}\right|$ on $L^{2}(\mu)$, and hence $S$ is a bounded operator on $L^{2}[0,1)$ as well.

Let $\lambda_{k}=e^{2 \pi i k \alpha} r^{\beta}$ for $k \in \mathbf{Z}$. By a theorem of Rudin [10], for each $N=$ $1,2, \ldots$ it is possible to choose a function $f_{N}$ continuous on the closed disk of radius $r^{\beta}$ and analytic on its interior such that

$$
\|f\|_{\infty} \leqq \sup _{k}\left|c_{k}\right|
$$

and $f_{N}\left(\lambda_{k}\right)=c_{k}$ for $|k| \leqq N$. For each $N$ choose a polynomial $p_{N}$ with

$$
\left\|p_{N}-f_{N}\right\|_{\infty}<1 / N \text {. }
$$

If $f \in L^{2}[0,1)$, write $f=\sum_{k=-\infty}^{\infty} a_{k} f_{k}$ in $L^{2}(\mu)$; then

$$
\begin{aligned}
& \left\|p_{N}(T) f-S f\right\|_{2}^{2} \leqq r^{2 n}\left\|p_{N}(T) f-S f\right\|_{L^{2(\mu)}}^{2} \\
& \quad=r^{2 n} \sum_{k=-\infty}^{\infty}\left|a_{k}\right|^{2}\left|p_{N}\left(\lambda_{k}\right)-c_{k}\right|^{2} \\
& \quad \leqq r^{2 n}\left[N^{-2}|| f \|_{L^{2}(\mu)}^{2}+\sum_{|k|>N}\left|a_{k}\right|^{2}\left(1+2 \sup _{j}\left|c_{j}\right|\right)^{2}\right] \rightarrow 0 .
\end{aligned}
$$

(3) We have seen already that there is $\gamma \in[0,1)$ such that $\tau x=\langle x+\gamma\rangle$ a.e. If we let

$$
\eta^{\prime}(x)=\psi(x)-\psi\langle x+\gamma\rangle
$$

then $(\mathrm{A})$ and $(\mathrm{C})$ imply that

$$
\eta^{\prime}(x)-\eta^{\prime}\langle x+\alpha\rangle=\eta(x)-\eta\langle x+\alpha\rangle \quad \text { a.e. , }
$$

and $\eta^{\prime}-\eta$ is constant a.e. Since (B) shows that $\arg \varphi(x)$ is also constant a.e., (3) follows.

(4) Routine verification using the computations found in the proof of (3).

Theorem 3.2. Suppose that $\beta \notin \mathrm{Z}_{\alpha}(\bmod 1)$ and let $S f(x)=\varphi(x) f(\tau x)$ $\left(f \in L^{2}[0,1)\right)$ be an invertible weighted translation operator, where $\varphi \in$ $L^{\infty}[0,1)$ and $\tau$ is a measure-preserving transformation on $[0,1)$. If $S T=T S$, then $S=c T^{n}$ for some $n \in \mathrm{Z}$ and some constant $c$.

Proof. We already know that $\tau x=\langle x+\gamma\rangle$ a.e. for some $\gamma \in[0,1)$. Since $S$ is invertible, $0<\|1 / \varphi\|_{\infty}<\infty$, and there is an $x_{0} \in[0,1)$ such that (C) holds and

$$
\|1 / \varphi\|_{\infty}^{-1} \leqq|\varphi(x)| \leqq\|\varphi\|_{\infty}
$$

for all $x \in\left\{\left\langle x_{0}+m \alpha+n \gamma\right\rangle: m, n \in \mathbf{Z}\right\}$. If we let

$$
N(n)=\sum_{k=0}^{n}\left[\chi_{[0, \beta)}\left\langle x_{0}+k \alpha\right\rangle-\chi_{[0, \beta}\left\langle x_{0}+\gamma+k \alpha\right\rangle\right],
$$

then $N(n)=\eta\left(x_{0}\right)-\eta\left\langle x_{0}+n \alpha\right\rangle$ for $n=1,2, \ldots$, and hence $N(n)$ is a boun- 
ded function of $n$. It follows then from a result of Furstenberg, Keynes, and Shapiro [3, Corollary 2.3] that $\gamma \in Z_{\alpha}(\bmod 1)$.

Suppose then that $\gamma=\langle n \alpha\rangle$ for some $n \in Z$ Z. If $n=0$, (B) and (C) imply that $\varphi$ is constant a.e. and so $S=c I$ for some constant $c$. If $n \neq 0$, we let

$$
g(x)=\left\{\begin{array}{rr}
\chi_{[0, \beta)}(x)+\chi_{[0, \beta)}\langle x+\alpha\rangle+\ldots+\chi_{[0, \beta)}\langle x+(n-1) \alpha\rangle & \text { if } n>0 \\
-\chi_{[0, \beta)}\langle x-\alpha\rangle-\chi_{[0, \beta)}\langle x-2 \alpha\rangle-\ldots-\chi_{[0, \beta)}\langle x+n \alpha\rangle & \text { if } n<0
\end{array}\right.
$$

and note that

$$
g(x)-g\langle x+\alpha\rangle=\chi_{[0, \beta)}(x)-\chi_{[0, \beta)}\langle x+n \alpha\rangle=\eta(x)-\eta\langle x+\alpha\rangle \text { a.e. }
$$

Since $g-\eta$ is invariant under translation by $\alpha$, it is constant and

But

$$
\varphi(x)=\operatorname{cr}^{g(x)} .
$$

$$
T^{n} f(x)=r^{g(x)} f\langle x+n \alpha\rangle \quad\left(f \in L^{2}[0,1)\right),
$$

and hence $S=c T^{n}$.

\section{Remarks and conjectures.}

The results of Section 3 extend to some other operators of the form

$$
T f(x)=r^{h(x)} f\langle x+\alpha\rangle \quad\left(f \in L^{2}[0,1)\right) .
$$

In particular, the analogue of Theorem 3.2 holds whenever the existence of an $L^{\infty}$ solution $\eta$ of

$$
\eta(x)-\eta\langle x+\alpha\rangle=h(x)-h\langle x+\gamma\rangle \quad \text { a.e. }
$$

implies that $\gamma \in Z_{\alpha}(\bmod 1)$. For some functions $h$, for example $h(x)=x$, the existence of a merely measurable solution of (D) implies that $\gamma \in Z_{\alpha}$ $(\bmod 1)$. It is likely that in Theorem 3.2 the hypothesis that $S$ be invertible is superfluous, and we conjecture that the existence of a measurable solution $\eta$ of $(\mathrm{C})$ already implies that either $\beta \in Z_{\alpha}(\bmod 1)$ or $\gamma \in Z_{\alpha}$ $(\bmod 1)$.

The proof of this conjecture would lead to some interesting results in the theory of diophantine approximations. For example, if we denote by $\|x\|$ the distance from a real number $x$ to the set of integers, then manipulation of Fourier series shows that (C) has an $L^{2}$ solution if and only if

$$
\sum_{k \neq 0} \frac{1}{k^{2}} \frac{\|k \beta\|^{2}\|k \gamma\|^{2}}{\|k \alpha\|^{2}}<\infty .
$$

Truth of the conjecture would then imply that this series converges if 
and only if either $\beta \in Z_{\alpha}(\bmod 1)$ or $\gamma \in Z_{\alpha}(\bmod 1)$. Indeed, it is reasonable to suppose that a series

$$
\sum_{k \neq 0} \frac{1}{k^{2}} \frac{\left\|k \beta_{1}\right\|^{2} \ldots\left\|^{\prime} k \beta_{n}\right\|^{2}}{\|k \alpha\|^{2}},
$$

where $\alpha$ is irrational, converges if and only if at least one $\beta_{i} \in Z_{\alpha}(\bmod 1)$.

We mention in conclusion a result along these lines that can be proved by the techniques of [8]. If $\alpha_{1}, \ldots, \alpha_{n}$ are rationally independent (in the sense that $\sum_{i=1}^{n} n_{i} \alpha_{i} \in Z$ for integers $n_{i}$ only if all the $n_{i}$ are 0 ), and if $\beta_{1}, \ldots, \beta_{n} \in[0,1)$, then

$$
\sum_{m_{i} \in Z-\{0\}} \frac{1}{m_{1}^{2} \ldots m_{n}^{2}} \frac{\left\|m_{1} \beta_{1}\right\|^{2} \ldots\left\|m_{n} \beta_{n}\right\|^{2}}{\left\|m_{1} \alpha_{1}+\ldots+m_{n} \alpha_{n}\right\|^{2}}<\infty
$$

implies that $\beta_{1} \ldots \beta_{n} \in \mathrm{Z}_{\alpha_{1}}+\ldots+\mathrm{Z}_{\alpha_{n}}(\bmod 1)$.

\section{REFERENCES}

1. J. J. Bastian, Decompositions of Weighted Translation Operators, Ph. D. Dissertation, Indiana University, 1973.

2. A. M. Davie, Invariant subspaces for Bishop's operators, Bull. London Math. Soc. 6 (1974), 343-348.

3. H. Furstenberg, H. Keynes and L. Shapiro, Prime flows in topological dynamics, Israel J. Math. 14 (1973), 26-38.

4. P. R. Halmos, Lectures in Ergodic Theory, Chelsea Publishing Co., New York, 1956.

5. S. Kakutani, Induced measure preserving transformations, Proc. Japan Acad. 19 (1943), 635-641.

6. A. Khintchine, Ein Satz Über Kettenbrüche, mit arithmetischen Anwendungen, Math. Z. 18 (1923), 289-306.

7. S. K. Parrott, Weighted Translation Operators, Ph. D. Dissertation, University of Michigan, 1965.

8. K. Petersen, On a series of cosecants related to a problem in ergodic theory, Compositio Math. 26 (1973), 313-317.

9. V. A. Rokhlin, $A$ "general" measure-preserving transformation is not mixing, Dokl. Akad. Nauk SSSR 60 (1948), 349-351 (in Russian).

10. W. Rudin, Boundary values of continuous analytic functions, Proc. Amer. Math. Soc. 7 (1956), 808-811.

11. J. Wermer, The existence of invariant subspaces, Duke Math. J. 19 (1952), 615-622.

UNIVERSITY OF NORTH CAROLINA

CHAPEL HILL, NORTH CARULINA, U.S.A. 